\title{
Environmental problems of using digital and information technologies in automobile transport
}

\author{
Yuri Trofimenko ${ }^{{ }^{*}}$ \\ ${ }^{1}$ Moscow automobile and road construction state technical university (MADI), 64, Leningradsky \\ prospect, Moscow, 125319, Russian Federation
}

\begin{abstract}
The use of digital and information technologies in automobile transport leads to the transformation and expansion of the environmental research problem field, because of the need to ensure sustainable development and to improve people's quality of life. It is necessary to learn how to manage the mobility (transport demand and transport behavior) of each resident in real time, offering him a choice of a limited set of transport behavior stereotypes. These stereotypes shall be based on the technologically sound code of ethics according to the need of ensuring the transport system environmental sustainability. The formation of a unified digital information environment for transport activities will allow the use of virtual and augmented reality technologies to manage population mobility by developing: systems for monitoring the impact of transport facilities on the natural and social environment; platform solutions of digital twins of roadside ecosystems, means of environmental engineering protection for use in digital models of road facilities, intelligent transport systems of traffic management; symbiotic man-machine management systems of transportation facilities. In case of the successful implementation of symbiotic man-machine management systems in the future transport facilities there are two ways for the motor-vehicle industry: either to create fully autonomous commercial transport, or to focus on symbiotic manmachine management systems of transportation facilities, which make the most of the driver's intellectual potential in decision-making. In our opinion, the second way is preferable in terms of safety, human movement comfort, habitat's quality of public transport. The first way seems to be more efficient and safer for freight transport.
\end{abstract}

\section{Introduction}

Problems of developing and using environmentally friendly, resource-efficient, energy efficient (low-carbon) vehicles, need to counter natural, man-made, biogenic, sociocultural threats, terrorism and extremism, cyber threats to transport systems and population, as well as search for effective answers to the challenges of interactions between man and nature, man and transport technologies can be successfully solved only by forming a unified information

* Corresponding author: author@email.org 
environment in automobile transport using big data processing technologies, machine learning and artificial intelligence [1].

Digital and information technologies change the vector of goal-setting of the automobile transport development. Accessibility, functional efficiency, environmental friendliness, safety of transport activities become the main factors of development, as conditions are created for rapid and comfortable movement and effective mobility of population through platform solutions within the unified digital information environment, "open" design and territorial planning, as well as improvement of vehicle designs, infrastructure, logistics technologies. At the same time, the factor of environmental sustainability, low-carbon development of transport is decisive for the medium term in most economically developed countries, including Russia [2, 3].

\section{Materials and methods}

The environmental consequences of the introduction of digital and information technologies in automobile transport, taking into account the change in the targets of its development, should be assessed in the light of the transformation and expansion of the problem field of environmental research, based on the need to ensure sustainable development, with the widespread use of traction electric motors of passenger and freight vehicles, buses, driverless vehicles [4]. At the same time, traditional forms of automobile transport influence on the natural environment will remain, as well as positive and negative impacts on the social environment of human habitat will increase. The main environmental challenges in the development of automobile transport over the medium term, in our opinion, will be:

- energy efficiency, carbon neutrality: minimizing consumption of traditional energy resources, greenhouse gas emissions;

- air pollution with fine suspended particles PM2.5, PM10, increased level of traffic noise, electromagnetic fields;

- insufficient security level of transport infrastructure facilities from natural, man-made and social emergencies, including cyber-attacks;

- alienation, aesthetic degradation of the territory: need to introduce biosphere-compatible and nature-like technologies of natural environment engineering protection, "green" architectural and planning design of the surrounding space using traditional, vertical, roof landscape gardening;

- impact on the social environment, human health, economic development of the territories;

- change in transport behavior motivation.

An adequate response to the above-mentioned challenges shall ensure the environmental sustainability of automobile transport development. Let us take a closer look at just one challenge that digital and information technologies have the greatest impact on. This is a change in the transport behavior motivation.

\section{Results}

Changing the transport behavior motivation under the influence of information and communication technologies is a new type of environmental impact that shall be regulated to achieve sustainable transport development. It seems that in order to eliminate traffic jams on the cities network of streets at the current or prospective level of motorization, even with fully autonomous vehicles, it is necessary to learn how to manage the mobility (transport demand and transport behavior) of each urban dweller in real time, based on the need for environmental sustainability, offering him a choice of a limited set of transport behavior stereotypes based on the technologically sound code of ethics. This is a difficult integrated 
environmental problem, which requires the adoption of agreed coordinating, information, organizational and advisory measures, formed with the involvement of a wide range of specialists, including mathematicians, sociologists, psychologists, economists, environmentalists, transport engineers, architects, IT specialists. To address this problem, it is necessary to carry out multidisciplinary studies on the transport technologies impact on the social environment (people's quality of life).

The formation of a unified digital information environment for transport activities will allow the use of virtual and augmented reality technologies to manage population mobility by developing:

- systems for monitoring the impact of transport facilities on the natural and social environment, including greenhouse gas emissions (energy consumption) in the full life cycle of transport facilities, as well as technical and operational and natural and climatic parameters for continuous forecasting of the risks of natural, man-made and social emergencies at transport infrastructure;

- platform solutions of digital twins of roadside ecosystems, means of environmental engineering protection for using in digital models of road facilities, intelligent transport systems of traffic management;

- symbiotic man-machine management systems of transportation facilities.

The development of man-machine management systems of transportation facilities, aimed at further transferring control functions from the driver to automatic devices, which can radically change the motivation of transport behavior, takes place in the following areas: intelligent man-machine interface; intelligent situational awareness; intelligent selfdiagnosis; smart management.

The creation of a single adaptive information control field in the vehicle (driver's cabin) is achieved: by the transition from large-format to seamless flexible indicators with high resolution; replacing two-dimensional (2D) images with three-dimensional ones (3D) using light field displays, stereoscopic, holographic and 3D visualization technologies; by the presence of indicators on the windshield, augmented and virtual reality systems formed inside and outside the vehicle.

Information input technologies are changing: sensory, voice, using analysis of oculomotor activity and gesture recognition, one-way and two-way neurointerfaces, in the future - by mind-reading. The driver perceives information with the use of hearing and visual sensors and tactors, taking into account his psychophysiological state. The driving algorithms adapt to the driver's needs and psychophysiological state.

Consider the implementation of some of these technologies in the design of the concept car of Mercedes Benz VISION AVTR 2020 [5].

This vehicle has a digital information communication space in which passengers interact with each other, with the vehicle and the environment and the principles of driving as a symbiotic man-machine system are implemented.

The vehicle is equipped with hundreds of different sensors connected to the intelligent system, which implement many functions of man-machine interaction. The vehicle "reacts" to the approach of the driver and passengers, visualizing the energy and information flow from them using digital neurons. Interaction between the driver and the vehicle takes place through the control unit: when the driver puts his hand on the central console, the cabin "comes to life", establishes a biometric connection with the driver. Visual communication between passengers and the environment is created by a curved display module. The environment around the vehicle (road, landscape) is displayed in real time in $3 \mathrm{D}$ graphics. The vehicle moves in driverless mode, but under the biometric control of the driver, which not only improves safety, comfort, but also forms a new quality of human habitat, changes the motivation of human's transport behavior. 


\section{Conclusions}

The use of digital and information technologies in automobile transport leads to the transformation and expansion of the environmental research problem field, because of the need to ensure sustainable development and to improve people's quality of life.

It is necessary to learn how to manage the mobility (transport demand and transport behavior) of each resident in real time, offering him a choice of a limited set of transport behavior stereotypes. These stereotypes shall be based on the technologically sound code of ethics code of ethics according to the need of ensuring the transport system environmental sustainability.

The formation of a unified digital information environment for transport activities will allow the use of virtual and augmented reality technologies to manage population mobility by developing: systems for monitoring the impact of transport facilities on the natural and social environment; platform solutions of digital twins of roadside ecosystems, means of environmental engineering protection for use in digital models of road facilities, intelligent transport systems of traffic management; symbiotic man-machine management systems of transportation facilities.

In case of the successful implementation of symbiotic man-machine management systems in the future transport facilities there are two ways for further technological development of the motor-vehicle industry: either fully autonomous commercial transport, or symbiotic manmachine management systems of transportation facilities, which make the most of the driver's intellectual potential in decision-making. In our opinion, the second way is preferable in terms of safety, human movement comfort, habitat's quality of public transport. The first way seems to be more efficient and safer for freight transport.

\section{References}

1. M. Gorodnichev, Y. Trofimenko, T. Potapchenko and L. Fedotova Intellectual analysis and estimation of gross greenhouse gas emissions //XII International Scientific Conference on Agricultural Machinery Industry/ IOP Conf. Series: Earth and Environmental Science 403012217 doi:10.1088/1755-1315/403/1/012217 (2019)

2. Y. Trofimenko, V. Komkov and V. Donchenko Problems and prospects of sustainable low carbon development of transport in Russia IOP Conference Series: Earth and Environmental Science 177(1), 012014 DOI:10.1088/1755-1315/177/1/012014 (2018)

3. Y. Trofimenko, V. Komkov, V. Donchenko and T. Potapchenko Model for the assessment greenhouse gas emissions from road transport Periodicals of Engineering and Natural Sciences 7, No. 1, June, pp.465-473 DOI: 10.21533/pen.v7i1. 390 (2019)

4. Y. Trofimenko, V. Donchenko and V. Komkov Methods and results of forecasting number and structure of motor fleet in the russian federation by types of engine and fuel used for calculation of greenhouse gases emission till 2050 International Journal of Emerging Trends in Engineering Research 8(6), pp 2707-2711, 79 DOI: 10.30534/ijeter/ 2020/79862020 (2020)

5. Inspired by the future: The VISION AVTR. URL 2020: https://www.mercedesbenz.com/en/vehicles/passenger-cars/mercedes-benz-concept-cars/vision-avtr/ 